\title{
Editorial: Seismicity in Volcanic Areas
}

\author{
Derek Keir ${ }^{1,2 *}$, Luca De Siena ${ }^{3}$, Cécile Doubre ${ }^{4}$, Jessica H. Johnson ${ }^{5}$, Francesco Maccaferri ${ }^{6}$ \\ and Luigi Passarelli ${ }^{7}$ \\ ${ }^{1}$ School of Ocean and Earth Science, University of Southampton, Southampton, United Kingdom, ${ }^{2}$ Dipartimento di Scienze della \\ Terra, University of Florence, Florence, Italy, ${ }^{3}$ Institute of Geosciences, Johannes Gutenberg University Mainz, Mainz, Germany, \\ ${ }^{4}$ Institut Terre et Environnement de Strasbourg, ITES, CNRS, ENGEES, Université de Strasbourg, Strasbourg, France, ${ }^{5}$ School of \\ Environmental Sciences, University of East Anglia, Norwich, United Kingdom, ${ }^{6}$ Vesuvius Observatory, National Institute of \\ Geophysics and Volcanology, Naples, Italy, ${ }^{7}$ National Institute of Geophysics and Volcanology, Bologna, Italy
}

Keywords: earthquake, volcano, tectonic, magma "mush", dyke, eruption, seismicity analysis

\section{Editorial on the Research Topic}

\section{Seismicity in Volcanic Areas}

Seismic activity (e.g., earthquakes, tremors) beneath volcanic areas is primarily caused by the dynamic interaction of molten rock and hydrothermal fluids with the solid host rock, by fracturing and fragmentation of the magma itself, and by tectonic processes interacting with the volcano. In addition, near-surface phenomena such as explosions and rockfalls at a volcanic edifice also produce seismic events. At volcano observatories globally, the real-time monitoring of the spatial and temporal patterns of seismic events is an essential geophysical tool to quantify the state of unrest, and forecast eruptions successfully. Seismic waveforms, earthquake catalogues and earthquake ray-path properties commonly supplement this tool to model the complex processes responsible for the earthquakes quantitatively, and to image subsurface magmatic and tectonic structures. Independent constraints provided by other disciplines such as geodesy and structural geology also significantly help scientists to understand the volcanic processes. Recent advances in earthquake recording technology, computing power and algorithms in artificial intelligence, allow processing and interpretation of large and complex multi-parametric datasets and scenarios.

"Our Seismicity in Volcanic Areas" research topic attracted 19 original research articles using seismic datasets to understand the processes acting in volcanic areas and their evolution in space and time. Such studies are critical for building a picture of how, when and where molten rock and volatile phases move and are stored in the crust, and how fluids interact with stressed and fractured rock. The result is an enhanced understanding of magmatic systems and more robust forecasts of future volcanic activity.

For this Editorial we divided the contributions to the research topic in five categories, which are articles that use: 1) Infrasound and seismic waveform data to constrain explosion related seismic sources; 2) Analysis and modelling of the space-time evolution of specific seismic swarms to understand the subsurface processes and triggering mechanisms causing them; 3) Spatially-large and/or temporarily-long earthquake catalogues to better understand subsurface magmatic processes and plumbing systems; 4) Earthquake tomography to image 3D variability of rock properties and interpret subsurface magmatic structures; 5) Automation of real-time analyses for large seismic datasets. A number of the studies in all of these themes also incorporate multi-disciplinary constraints to aid the interpretation of seismic results.

Seismology and infrasound are among the most popular techniques for monitoring volcanoes since they offer an excellent temporal resolution of subsurface and near-surface earthquakes and eruptive activity. However, a key element of volcano monitoring is understanding what magmatic and volcanic processes are responsible for seismic and acoustic signals observed. Diaz-Moreno et al. present the first comprehensive observational baseline characterization of acoustic signals at Volcán de Fuego in Guatemala. They find that acoustic signals are dominantly short-duration acoustic 
transients linked to discrete ash-rich and gas-rich explosions, and other indirect volcanic processes such as lahars. They also find more sustained seismo-acoustic tremors and chugging emissions likely sourced from pressurized magma and gas and explosions. Iezzi et al. and Rohnacher et al. use both seismic and infrasound data to quantitatively model source characteristics of seismoacoustic signals associated with explosions at Mount Cleveland in the United States, and Santiaguito volcano in Guatemala, respectively. Iezzi et al. identify time lags in the arrival time of the acoustic and seismic signals associated with explosions. Using a combination of waveform cross-correlation and analysis of particle motion dip angles, they show that a varying explosion source depth between 1.5 and $2 \mathrm{~km}$ within the conduit likely plays a dominant role in the observed variations in time lag. Rohnacher et al. constrain source mechanisms and depths of seismic signals leading up to explosions using array and particle motion analyses. Results suggest the seismic signals are caused by the opening of tensile fractures at a few hundred meters depth followed by rapid gas ascent leading to the explosion.

Earthquake swarms in volcanic areas carry a wealth of information on their causative processes. Spatial and temporal evolution of earthquake hypocentres and their source characteristics can be diagnostic on whether earthquake swarms are caused by magmatic intrusion or due to aseismic processes (i.e., pore-pressure diffusion and aseismic slip) facilitated by crustal fluid redistribution. However, independent observations such as deformation and geophysical data, modelling processes along with consideration of tectonic setting are essential to single out the triggering mechanisms of seismicity in volcanic areas. Seven contributions of this research topic clearly represent good practice of using multiple complementary datasets to eventually understand the nature of seismic activity. For example, fast migration velocity of accurately located earthquake hypocentres and normal faulting earthquakes inferred by Benson et al. indicate that the 2019 earthquake swarm underneath Tarawera volcano, New Zealand, is the seismic signature of a dike intrusion at the brittle-ductile transition. During the 2015 earthquake swarm at Sakurajima volcano, Japan, Midori and Nakamichi interpret the spatial distribution of precisely located earthquakes and the temporal changes in their source mechanism as caused by local stress changes imparted by a fast inflating dike intrusion. Bonaccorso and Giampiccolo successfully apply an equation that links the seismic energy associated to dike-induced earthquakes to the geodetically inferred dimension of the 2018 complex dike intrusion at Mt. Etna, Italy. Future fine tuning of this equation could eventually allow the magnitude of radiated seismic energy to be used as a proxy for the size of dike intrusions in early warning applications.

The analysis of earthquake catalogues combined with thermorheological modelling constrained by various geophysical observations at the Main Ethiopian Rift performed by Muluneh et al. indicate lower-crust earthquake swarms sourced from redistribution of crustal fluids. The authors focus on differences in lower crustal seismicity directly beneath, and outboard the rift valley, and estimate lower crust permeability values. They inferred that pore-pressure diffusion processes are the driving mechanism for the observed swarm-like seismicity. Sahara et al. revise the locations of almost 3,000 VT seismic events that occurred during nearly 6 weeks prior the November 21, 2017 Agung volcano eruption (Bali, Indonesia). The authors reconstruct the complex dynamics of magma rising through the plumbing system, and conclude that the intrusion was temporarily arrested by a lithological discontinuity at around $6 \mathrm{~km}$ depth. For the same eruption, Wellik et al. focus on seismic rate variations and repeating earthquake families showing that the evolution of earthquake families reveal the progression toward eruption even though the overall earthquake rates and seismic energy release declined. Liu et al. use template matching techniques on micro-seismic events recorded by a local station near Changbaishan volcano on the China and North Korea border. Their analysis suggests that during the 2002-2005 unrest-which was triggered by a large regional earthquake - a swarm of VT events was caused by deep magma degassing that promoted a small magmatic injection to a shallower reservoir at about $5 \mathrm{~km}$ depth.

Long time series of seismological measurements have led to highly accurate depictions of the evolution of seismic activity affecting volcanic areas. These have helped to elucidate the processes that take place at depth, and in particular the transfer of fluids towards the surface. A challenge with this type of analysis is to properly visualize the hypocentral data in space and time, and with respect to other datasets. Guardo et al. propose a method based on a Machine-Cube algorithm to identify seismic and aseismic volumes below the Etna edifice. Using previous tomographic images and modelled deformation sources deduced from surface measurements, they refine the $3 \mathrm{D}$ image of the magma storage and explain the deep instability below the eastern flank by both the magmatic and gravitational origins. The re-evaluation of large seismic catalogues acquired over a long time period allows a wide overview on the evolution of the volcanoes themselves and on their main seismogenic structures required for risk assessment. For the Campanian volcanoes in Southern Italy, Giudicepietro et al. point out the role of hydrothermal activity in the recent increase of seismicity in this highly populated region.

In rifting context, the co-existence of tectonic-regional and magmatic-local stress fields remains the main challenge to understanding the origin of both the background seismicity and the occurrence of swarms. In the Natron rift of the continental rift of East Africa, a dense temporary network has allowed a large seismic catalogue of hypocentres and focal mechanisms to be established. Reiss et al. separate tectonic and volcano-induced stresses to demonstrate the role of volcanic activity on the transfer of deformation from border faults to the centre of the magmatic rifts. In a more evolved rift setting, the 60-year-long compilation of catalogues in the northernmost East African rift by Ruch et al. revealed unprecedented details of the seismic swarms episodically affecting the same areas, and which have mainly been induced by transient magma supply at depth. Finally in oceanic context, Einarsson and Brandsdottir present a catalogue of 50 years of 
monitoring in Northern Iceland, offering an extremely precise picture of the dyking sequence in the Krafla and adjacent segments. The time-space evolution of the seismicity, together with surface deformation data and geophysical investigations, are crucial for depicting the processes involved in the creation of oceanic crust.

Localizing earthquakes requires the assessment of the velocity structure of the volcano. A resolved $3 \mathrm{D}$ velocity model can dramatically improve the accuracy of seismic locations, while the comparison between locations and velocity anomalies helps clarify the magmatic, hydrothermal or tectonic origin of seismicity. Wilks et al. present the first velocity and velocityratio model of Aluto volcano (Ethiopia), obtained using traveltime tomography based on grid-based eikonal solvers and probabilistic location algorithms. The study shows sufficient resolution to distinguish shallow hydrothermal and deeper magmatic systems and detect their connection. Ardianto et al. use a similar combination of travel-time tomography and probabilistic location to resolve velocity and velocity ratios at Mt. Agung volcano (Indonesia), using data recorded around its 2017 eruption. The study highlights an aseismic zone of low velocity and high velocity ratios, from which magma could have migrated sub-vertically to feed the eruption. Seismicity also clusters in a region of moderate-to-high ratios, interpreted as a sub-vertical dyke complex interacting with the local fault network.

The recognition of earthquake and tremor characteristics and the ability to track their migrations in space and time are becoming standard practice; however, when automatized and applied in real-time, these techniques acquire unprecedented value for volcano monitoring institutions and eruption forecasting. Cortés et al. propose an automatic VolcanoSeismic Recognition system trained using data collected at global volcanoes, to overcome the lack of unified criteria for earthquake characterization. The four example applications show the recognition potential of the tool, especially for volcano observatories lacking a working recognition system. Smith and Bean develop a REal-Time TREmor Analysis Tool (RETREAT) comprising advanced array-analysis techniques for the real-time detection and characterization of tremors. Their two example applications demonstrate the flexibility of the web-based interface at the scales of the European Arctic and Bárðarbunga (Iceland) dike intrusion.

\section{AUTHOR CONTRIBUTIONS}

DK, LS, CD, JJ, FM, and LP edited the Research Topic "Seismicity in Volcanic Areas." All the authors listed have made a substantial direct and intellectual contribution to the work and approved it for publication.

\section{FUNDING}

The Research Topic Editorial activities of DK were supported in part by the Ministero dell'Universitàe della Ricerca (MiUR) through PRIN grant 2017P9AT72. The Research Topic Editorial activities of LS were supported by the Terrestrial Magmatic Systems Research Platform, a high-potential research initiative of the universities of Mainz, Frankfurt and Heidelberg partially funded by the state of Rheinland-Pfalz. LP acknowledges funds of NEWTON-g project grant agreement no. 801221.

\section{ACKNOWLEDGMENTS}

We thank the authors for their contributions, and the reviewers and editorial staff for their efforts to pull together this article collection. We also thank the Editorial Office of Frontiers in Earth Science for the kind invitation to edit this Research Topic and editor in chief VA for his support.

Conflict of Interest: The authors declare that the research was conducted in the absence of any commercial or financial relationships that could be construed as a potential conflict of interest.

Publisher's Note: All claims expressed in this article are solely those of the authors and do not necessarily represent those of their affiliated organizations, or those of the publisher, the editors and the reviewers. Any product that may be evaluated in this article, or claim that may be made by its manufacturer, is not guaranteed or endorsed by the publisher.

Copyright (C) 2021 Keir, De Siena, Doubre, Johnson, Maccaferri and Passarelli. This is an open-access article distributed under the terms of the Creative Commons Attribution License (CC BY). The use, distribution or reproduction in other forums is permitted, provided the original author(s) and the copyright owner(s) are credited and that the original publication in this journal is cited, in accordance with accepted academic practice. No use, distribution or reproduction is permitted which does not comply with these terms. 